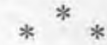

Tout cet ensemble, rejoignant d'importantes eonsidérations économiques que nous n'avons pu aborder ici, nous parait montrer à quel point s'impose une révision profonde de nos actuelles conceptions et dispositions réglementaires sur le problème capital qu'est celui de l'approvisionnement en lait des populations, selon les diverses destinations de cet aliment direct de valeur primordiale.

\title{
LA RECHERCHE CHIMIQUE ET L'INDUSTRIE DU LAIT
}

Au dernier Congrès de l'American Chemical Society, (automne 1947), G. S. Supplee de la G. C. Supplee Research Corporation, a prononcé une remarquable conférence sun l'application de la recherche chimique dans l'industrie du lait, communication qui a été considérée comme une des plus intéressantes du Congrès et qui à ce titre a reçu le Prix Borden attribué à l'auteur d'une étude sur l'industrie laitière. Notre Collaborateur, M. G. GÉnin, Ingénieur E. P. C., a reproduit ci-dessous les points essentiels de cette conférence qui fixe les questions sur lesquelles devront porter dans le proche avenir tous les efforts des chercheurs.

G. THEULin.

Le lait est la matière essentielle qui alimente une vaste industrie intéressant à la fois des millions de producteurs et des consommateurs encore plus nombreux. Le lait, comme tous ses sousproduits, est une denrée essentiellement périssable. C'est en outre une substance alimentaire capable de servir de support aux agents de transmission de nombreuses maladies et à ce titre, sa production, son traitement et sa distribution ont nécessité l'établissement de règlements destinés à assurer la protection du public contre le transfert de maladies, ou simplement contre l'absorption d'un produit défectueux. Aujourd'hui, le contrôle bactériologique et sanitaire du lait est entré dans nos mœurs et toute violation des règles qui précisent ce contrôle ne serait tolérée ni par les organismes chargés de la surveillance de cette denrée, ni même par le public lui-même.

Le sujet de cette étude porte toutefois plus sur les questions liées à la chimie du lait que sur celles qui interviennent dans son contrôle sanitaire. Le lait qui constitue un ensemble particulièrement hétérogène de substances nutritives est créé par la nature pour un but bien déterminé, l'élevage des jeunes êtres et ni le chimiste, ni l'ingénieur n'ont été capables pendant longtemps d'améliorer ce produit en en modifiant les caractéristiques ou de le produire par synthèse. 
Il s'est créé ce qu'on appelle aujourd'hui une branche de la chimie dite chimie du lait. En réalité, on doit reconnaître que cette branche de la science moderne est constituée par des domaines très enchevêtrés qui intéressent plusieurs sections de la chimie et de la biologie. Les objectifs que l'on eherche à atteindre lorsque l'on entreprend des recherches sur le lait sont multiples. Certains sont purement utilitaires, d'autres posent des problèmes de recherche pure. On se propose, par exemple, par des déterminations analytiques, d'identifier et de doser quantitativement l'ensemble des constituants du lait qui comprennent parfois des traces de substances organiques ou inorganiques dont la présence dans le lait ne semble jusqu'à aujourd'hui que jouer un rôle absolument futile et purement académique.

Cependant, lorsque l'on passe en revue les travaux déjà entrepris sur le lait ou sur ses dérivés, on constate que des recherches antérieures qui paraissaient sans objet immédiat ont subi une véritable évolution et ont conduit à des constatations présentant une importance beaucoup plus grande que celle sur laquelle on comptait initialement. C'est par exemple le cas des recherches entreprises entre 1919 et 1922 sur la présence du cuivre dans le lait qui ont conduit à des conclusions fondamentales sur le rôle essentiel que peuvent jouer des traces minimes de ce métal dans la détérioration du lait. De même, au cours des quinze années qui ont suivi la découverte de la lécithine dans le lait, découverte qui date de 1900 , on ne se rendait pas compte de l'importance de la présence de ce produit dont un des constituants, la choline, par sa décomposition en triméthylamine contribue au goût de poisson qui apparaît dans certains beurres défectueux et qui pendant des dizaines d'années a été le cause de pertes très importantes subies par l'industrie laitière. Rappelons également que l'on croyait sans intérêt la présence du 7-déhydrocholestérol dans le lait jusqu'au jour où l'on a constaté que c'est ce produit qui constitue la substance active antirachitique du lait, substance qui se transforme en vitamine $\mathrm{D}_{1}$ lorsque le lait est exposé à l'action des rayons ultraviolets. On pourrait eiter de nombreux autres exemples de recherches initialement sans intérêt pratique et qui, par la suite, se sont trouvés au contraire être le point de départ d'applications essentielles dans le domaine de la chimie laitière.

Cependant, le plus fréquemment, les recherches sont dirigées vers des objets bien définis, présentant un intérêt utilitaire immédiat. La mise au point aujourd'hui si fouillée de la méthode de S. M. Babeock pour le dosage de la graisse du lait, constitue un cas partieulier d'une étude entreprise dans un but utilitaire et parfaitement déterminé. L'essai Babcok constitue un exemple de 
recherche ehimique spéciale à l'industrie latière et qui certainement a contribué à fixer dans le monde entier certains aspects de l'économie de cette industrie.

Le lait contient plus de $85 \%$ d'eau ; e'est une substance éminemment périssable. Il est produit dans des millions d'établissements isolés, distribués dans le monde rural, et dans lesquels il est impossible d'établir une surveillance analogúe à celle que l'on peut aujourd'hui réaliser dans un établissement industriel. Ces conditions particulièrés, ont conduit, au début de ce siècle, à la généralisation de pratiques frauduleuses parmi lesquelles l'addition d'eau au lait ou l'addition d'agents conservateurs, étaient les plus répandus. Pendant longtemps, les recherehes dans le domaine du lait ont été concentrées sur la mise au point de méthodes analy. tiques permettant de lutter eontre de telles méthodes frauduleuses et aujourd'hui encore, les recherches se poursuivent en vue de mettre au point une interprétation judicieuse des résultats des déterminations analytiques.

L'emploi du lait et d'une façon générale des produits laitiers comme produits alimentaires constitue un héritage et une tradition de la race humaine. Néanmoins, les progrès qui ont été récemment réalisés dans le domaine de la nutrition ont modifié profondément notre opinion sur l'intérêt du lait et de ses dérivés. Initialement, le chimiste s'était efforcé de déterminer par analyse la composition brute du lait; il déterminait par exemple sa teneur en eau, en protéines, en graisse, en matières minérales et en hydrates de carbone. Puis le physiologiste est intervenu et rapidement, il a établi un nouveau programme de recherches ayant pour objet d'établir une relation entre les constituants du lait et les exigences alimentaires de l'organisme de l'homme et des animaux. Ces recherches ont tout d'abord porté sur la teneur en calcium et en phosphore du lait et on a constaté que les substances minérales contenues dans le lait jouaient un rôle essentiel dans le métabolisme minéral de l'homme. C'est alors que l'on a essayé d'établir quels pouvaient être les besoins de l'homme en calcium pour le mener de l'enfance à son état adulte. Un autre exemple de ces recherehes initiales dans le domaine de la physiologie nous est donné par l'étude des variations qualitatives des diverses protéines contenues dans les aliments et ce sont des recherches de ce genre qui ont permis d'établir la supériorité au point de vue nutritif des protéines du lait sur les propriétés d'autres sources.

C'est alors que sont apparus deux nouveaux types de recherches portant sur le lait et les produits laitiers, à la suite de ce que l'on a pu appeler l'avènement de l'ère des vitamines. La déeouverte des vitamines a considérablement reculé l'horizon jusqu'alors borné 
des chimistes de l'alimentation, en révélant l'existence dans ces produits d'une puissance qui a profondément bouleversé l'imagination du chimiste et dú biologiste. Il était naturel que le lait, en tant que fluide physiologique, constituât un excellent champ d'investigation pour l'étude de ces nouveaux produits et il n'est pas étonnant que toutes les vitamines connues jusqu'à aujourd'hui aient été décelées en plus ou moins grandes quantités dans le lait. Les recherches nombreuses et les déterminations faites sur la teneur en vitamines du lait n'ont d'ailleurs pas facilité la tâche de l'industrie. On a en effet constaté assez rapidement que la teneur en vitamines du lait pouvait varier d'une façon très sensible et que par exemple le caractère irrégulier de la nourriture donnée aux animaux ou les différents traitements subis par le lait, pouvaient entraîner pour ce produit des variations considérables de son pouvoir nutritif qui auparavant n'avaient jamais été suspectées.

Un des problèmes qui s'est alors posé à l'industrie a été d'enrichir le lait en certaines vitamines et en particulier en vitamine $D$ dont il ne contient pas de grandes quantités. Le rôle du lait dans l'alimentation infantile et la lutte contre le rachitisme des enfants, a entraîné un développement immédiat des méthodes d'enrichissement du lait, surtout à partir du jour où l'on a constaté qu'il était possible d'enrichir le lait en vitamine D par action de la lumière ultraviolette. Très rapidement, on est parvenu à livrer à la consommation des laits enrichis en vitamine $\mathrm{D}$, soit comme il y a vingt ans, en ajoutant à ce lait de la levure irradiée ou en le soumettant directement à l'action des irradiations, soit plus récemment, en lui ajoutant différents concentrés de vitamine. Cet enrichissement du lait en vitamine D a constitué une date capitale dans l'histoire du lait, car pour la première fois, la science permettait, par son esprit créateur, de modifier et d'enrichir les propriétés nutritives d'un produit, qui jusqu'alors avait été considéré comme devant avoir une composition inaltérée et déterminée par la nature.

En ce qui concerne la vitamine A, la richesse relative du lait en cette vitamine confère au lait et à ses dérivés un rôle essentiel en tant que produit nutritif. C'est alors qu'on a constaté que cette teneur en vitamine pouvait dépendre de la race des vaches laitières et du caractère de leur alimentation. Une telle découverte a conduit à un résultat qui n'avait pas été initialement envisagé et qu'il était difficile de prévoir, résultat qui s'est manifesté par l'incorporation dans un des produits les plus dangereux au point de vue économique pour le beurre : la margarine, de vitamine A ou de produits précurseurs de la vitamine $\mathrm{A}$, de façon à donner à cette margarine les qualités nutritives du beurre. 
Aujourd'hui, on a reconnu le rôle essentiel que joue la compo, sition de la nourriture donnée aux animaux dans la fixation de la valeur nutritive du lait et en particulier sa teneur en vitamines et en certains constituants inorganiques, souvent présents à l'état de traces. On a done été conduit à approfondir eette question, afin d'améliorer la qualité nutritive du lait et de régulariser ses variations saisonnières. C'est ainsi par exemple que la teneur en iode du lait qui, normalement, ne représente que quelques parties par milliard de parties de lait est considérablement influencée par la teneur en iode de l'alimentation donnée au bétail. Il n'a pas encore été démontré que l'on pourra maintenir une uniformité constante de la composition du lait, mais par exemple on peut espérer par un contrôle de la nourriture du bétail lutter par exemple plus efficacement contre la carie dentaire, car l'expérience a montré que le lait fourni par des animaux dont l'alimentation provient de sols riches en fluor a une teneur légèrement plus élevée en fluor qu'un lait normal. Il reste néanmoins à établir d'ûne façon définitive les relations exacts qui existent entre la composition du lait et la nourriture du bétail.

A ce point de cet exposé, il est intéressant de rappeler le fait que de nombreuses substances inorganiques ont été décelées à l'état de traces dans le lait, eertaines d'entre elles ne pouvant être précisées que par des méthodes spectrographiques. Etant donné qu'on a déjà constaté le rôle important que peuvent jouer des traces de fer ou de cuivre dans le lait, il n'est pas ridicule de penser que certains autres constituants peuvent jouer un rôle tout aussi considérable.

En ce qui concerne par exemple le fer, on a constaté que dans un lait parfaitement pur et non contaminé, il peut être présent dans la proportion de une à deux parties par million de.lait. Or l'effieacité de ce produit dans l'alimentation humaine peut être réduit par la présence de phosphates eontenus également dans le lait et cela pourrait expliquer l'anémie qui peut survenir chez un individu dont l'alimentation est uniquement à base de produits laitiers. On sait également que le cuivre contenu dans le lait, même dans une proportion inférieure à 1 millionième, cuivre qui dans ce cas provient presque toujours d'une contamination du produit, pose de très importants problèmes dans tous les cas de fabrication de dérivés du lait, car ce produit exeree un effet catalytique considérable sur l'oxydation du lait-liquide, du lait en poudre et du beurre. La découverte de ce fait a entrainé de profonds bouleversements dans le choix des matériaux utilisés par exemple pour la construction de l'appareillage. Ces quelques exemples illustrent donc parfaitement les conséquences considérables qui ont découlé pour l'industrie 
laitière des recherches dynamiques et extraordinairement fécondes de la chimie alimentaire.

Aujourd'hui, la science de l'alimentation a fait des progrès considérables et on commence à connaître ce que doit être une ration bien équilibrée. Ce sont les recherches dans ce domaine qui ont montré aux spécialistes le rôle prédominant que peut jouer le lait parmi les produits alimentaires. Il n'est done pas étonnant que l'on s'efforce continuellement d'améliorer la qualité nutritive du lait, de maintenir et d'accroître ses qualités organoleptiques, d'éviter sa détérioration, de façon à répondre toujours plus complètement aux exigences du consommateur. Aujourd'hui, il apparaît qu'on ne doit plus se contenter de consommer le lait tel qu'il est fourni par l'animal, mais qu'on doit s'efforcer d'en améliorer la composition, la stabilité, soit par une meilleure alimentation du bétail, soit par des traitements mieux appropriés, soit même par des additions appropriées de substances étrangères.

Il est certain que toutes les recherches portant sur l'amélioration des propriétés organoleptiques, la meilleure conservation du lait et l'utilisation plus rationnelle des sous-produits de l'industrie laitière, sont d'une application plus immédiate que les travaux de longue haleine qui portent sur l'amélioration des qualités nutritives du lait. Toutefois, les techniques et les procédés qui sont aujourd'hui couramment utilisés dans la fabrication des dérivés du lait ont souvent fait leur apparition à là suite d'une recherche purement spéculative qui avait révélé incidemment le rôle important joué par telle ou telle impureté du lait, Cette observation s'applique partieulièrement à la fabrication de certains produits, comme par exemple le lait en poudre et le lait concentré. Par ailleurs, la centralisation de la fabrication du beurre qui de plus en plus se fait dans des usines et de moins en moins dans les fermes, a permis d'améliorer la qualité et l'uniformité de ce produit. Enfin, les progrès constants qui ont été apportés à la fabrication du fromage résultent également, pour la plupart d'entre eux, de travaux de recherches entrepris sur la chimie du lait.

L'oxydation est un des défauts essentiels qui se produisent dans les produits laitiers. C'est par exemple cette oxydation qui est respónsable de la saveur défectueuse que prend le lait conservé pendant vingt-quatre heures. C'est également l'oxydation qui est responsable de la destruction d'une proportion substantielle de l'acide ascorbique contenu dans le lait, destruction qui se produit très souvent avant même que le lait ait pu être distribué au consommateur. Les recherches d'ordre chimique entreprises en vue de connaître plus complètement le mécanisme des réactions qui se produisent dans ces différents cas, ont mis en évidence l'action 
catalytique de traces infimes de cuivre, la relation qui existe entre la destruction de l'acide ascorbique et l'apparition de goût d'oxydation et enfin, le rôle éminent du potentiel d'oxydation-réduction. C'est à partir du moment où tous ces faits ont été bien, reconnus que l'on a pu découvrir le rôle essentiel de l'oxygène dissous dans le lait. C'est alors que l'on a mis au point des méthodes de traitement pouvant être utilisées dans de grandes installations et eapables de faire disparaître les dernières traces de l'oxygène dissous dans le Jait. On a constaté qu'en prenant eette précaution, il était possible de supprimer complètement l'apparition des goûts défectueux dans le lait et de maintenir sa teneur en vitamine C. Ces mêmes techniques sont en voie d'être appliquées à la fabrication du lait concentré stérilisé.

Si le lait en poudre est relativement stable lorsqu'on le compare an lait liquide, il est néanmoins susceptible de s'oxyder spontanément, en particulier ses constituants gras. D'importantes recherches ont été entreprises sur les différentes étapes de l'oxydation des graisses. On a par exemple étudié la période d'induction, les phénomènes d'auto-oxydation et les phénomènes catalytiques qui interviennent dans cette réaction. C'est à la suite de ces recherches qu'a été imaginée la méthode de conservation du lait en poudre dans un gaz inerte. Cette pratique, mise au point industriellement pour la première fois en 1924 a permis d'assurer la distribution dans le monde entier de poudre de lait entier, chose qui était absolument impossible auparavant, par suite d'une oxydation rapide de la graisse.

Le temps ne nous permet pas de passer en revue tous les travaux qui ont permis à l'industrie laitière de réaliser d'importants progrès au cours de ces dix dernières années, ou qui vont permettre à cette industrie de faire de nouveaux pas en avant. Quelques exemples montreront néanmoins la diversité des traivaux actuellement entrepris :

Détermination de la teneur limite en humidité de la poudre de lait au-dessous de laquelle il faut la maintenir pour prolonger les qualités de conservation du produit.

Irradiation directe du lait pour la synthèse de la vitamine D par application instantanée de rayons ultraviolets de forte intensité.

Découverte de la stabilisation de la vitamine $\mathrm{D}$ par les protéines dispersées du lait.

Nouveau procédé de fabrication du fromage par l'emploi de sels peptisants.

Pasteurisation rapide à haute température du lait liquide et application de cette même technique à la stérilisation du lait concentré. 
Découverte du rôle important que joue l'équilibre entre les constituants minéraux du lait sur la coagulation et les propriétés physiques du lait conservé.

Rôle des enzymes lypolytiques sur la détérioration des différents produits laitiers.

Recherches ayant contribué à l'amélioration de la qualité et des possibilités de conservation du beurre.

Découverte de la riboflavine dans le lait et dans le sérum et rôle important joué par cette substance dans l'alimentation.

Découverte d'un produit encore non identifié qui existe dans la portion dégraissée du lait et qui constitue un stimulant très puissant de la croissance.

Etude analytique et clinique des propriétés nutritives de la caséine et de la lactalbumine et des emplois thérapeutiques d'hydrolysats de ces produits.

Peut-être que certains de ces travaux ne conduiront pas à des conclusions utiles pour l'industrie laitière, l'avenir seul permettra de faire la distinction entre les résultats utiles et ceux ne présentant plus d'intérêt. C'est ainsi par exemple que les très importantes recherches sur l'irradiation directe du lait sont devenues aujourd'hui sans objet. On est parvenú en effet à enrichir le lait en vitamine D par l'addition de concentrés d'une façon beaucoup plus économique et plus simple que par les méthodes reposant sur l'irradiation. La synthèse à laquelle on est parvenu de la riboflavine diminue l'intérêt que présentait le lait comme source riche en riboflavine, car la riboflavine synthétique est aujourd'hui fabriquée à faible prix. De même, la rapidité avec laquelle on met actuellement au point la synthèse des amino-acides essentiels à l'alimentation de l'homme, pourra dans un proche avenir modifier la position des protéines contenues dans le lait.

Les travaux entrepris sur le lait et ses dérivés sont périodiquement influencés par la situation économique de la production soumise à des périodes de surproduction. A chaque eycle, les recherches ayant pour objet de trouver de nouveaux emplois pour le lait et ses dérivés, reçoivent de ce fait une nouvelle impulsion. On. s'efforce done de trouver des méthodes permettant, en partant de ces surplus d'augmenter la production de lait en poudre, de beurre, de fromage, mais on s'efforce également de trouver des applications entièrement nouvelles, non seulement pour le lait, mais également pour tout ce qui constitue les sous-produits de l'industrie laitière.

On sait aujourd'hui que le lait écrémé peut constituer le point de départ, ou encore la matière première d'un grand nombre de substances qui touchent plus l'industrie chimique proprement dite que l'industrie alimentaire. A ce stade, le lait écrémé a perdu 
totalement son individualité en tant que produit servant à l'alimentation. Rappelons en effet que l'on peut préparer, en partant de lait écrémé, la caséine industrielle, le sérum que l’on peut dessécher en vue de l'utiliser pour l'alimentation du bétail, ou en vue d'en extraire la lactalbumine, le sucre de lait, les protéines du lait non coagulables et enfin les phosphates organiques et inorganiques. Les eaux-mères provenant de la fabrication du sucre de lait peuvent être elles-mêmes employées comme source de substances minérales solubles ou de composés encore inconnus qui pendant un certain temps ont été utilisés comme point de départ de la fabrication de la riboflavine cristalline naturelle.

La fabrication de la caséine pour des applications industrielles était déjà généralisée dans certaines contrées, bien avant que l’on connaisse les propriétés nutritives de ce produit. La caséine sert aujourd'hui de point de départ pour la fabrication des produits industriels tels que colle, matières plastiques, peintures, et plus récemment, on l'a utilisée dans la fabrication des fibres textiles. Diverses matières organiques ou inorganiques peuvent être mélangées à la caséine, ou réagir avec elles et modifier d'une façon. importante ses propriétés physiques, en vue d'en faciliter les emplois. Néanmoins, par suite de la pénurie mondiale des produits alimentaires et à la suite de travaux qui ont montré la place importante que peut jouer la caséine comme protéine dans l'alimentation humaine, il s'est établi une concurrence de plus en plus accentuée, entre les emplois de la caséine comme matière première et ses applications comme produit alimentaire.

La cáséine est la première substance que l'on extrait du lait écrémé lorsque l'on cherche à utiliser complètement ce produit. Il reste alors, après séparation de la caséine, un sérum qui est la matière première de départ pour la fabrication du lactose qui représente environ $75 \%$ des substances solides contenues dans ce sérum. L'extraction du lactose à différents états de pureté est une industrie déjà établie aux Etats-Unis depuis environ soixante ans. Un des plus importants progrès dont a bénéficié cette industrie, au cours de cette période, a été le perfectionnement des méthodes de production industrielle d'un type de lactose très soluble, appelé le lactose $\beta$ alors que la forme plus courante de lactose est l'hydrate $\alpha$. Actuellement, il est fabriqué chaque année des quantités importantes de lactose $\beta$ à $100 \%$ de pureté, alors qu'il y a encore quelques années, ce produit était simplement une curiosité de laboratoire.

A côté du lactose, le sérum renferme de la lactalbumine, des substances protéiniques non identifiées et qui renferment en particulier les lypoprotéines du lait, des enzymes, des vitamines solubles 
dans l'eau, des substances azotées de faible poids moléculaire et enfin pratiquement, tous les constituants minéraux du lait. La lactalbumine peut être facilement coagulée et séparée quantitativement du sérum en réglant le $p H$ à une valeur appropriée et sans qu'il soit nécessaire d'ajouter des agents de précipitation. La lactalbumine, ainsi séparée, additionnée ou non d'autres substances convient, comme point de départ, pour la fabrication de produits thérapeutiques et d'aliments spéciaux et ses emplois se sont très développés depuis quelques années. Pappelons que les lipides contenus dans cette fraction renferment environ $70 \%$ de matières insaponifiables, en grande partie du cholestérol, fondant entre 147 et $148^{\circ} \mathrm{C}$.

Les recherches qui ont été entreprises en vue de l'utilisation des eaux résiduaires et des eaux-mères provenant de la fabrication du sucre de lait avaient déjà montré que ce produit favorisait la eroissance, en particulier lorsqu'on l'additionnait de substances extraites du riz. A cette époque, on ne connaissait que peu la question des vitamines et on faisait difficilement la distinction entre vitamines solubles dans les graisses et vitamines solubles dans l'eau. L'évolution de nos connaissances sur les vitamines a permis de montrer que les mélasses provenant de la fabrication du sucre de lait renferment de nombreuses vitamines solubles dans l'eau, la riboflavine étant un des plus importants de ces produits. C'est vers 1935, que la fabrication industrielle de riboflavine cristalline naturelle extraite des eaux-mères du sucre de lait a été mise au point et pendant plusieurs années, ces eaux-mères ont été le seul produit servant à la fabrication de la riboflavine pure. Cette situation enviable ne s'est malheureusement pas prolongée et aujourd'hui, l'ingéniosité des chimistes est parvenue à mettre assez rapidement au point des méthodes de synthèse de la riboflavine permettant une fabrication en grande quantité et économique de ce produit.

La chimie ayant déjà réalisé de grands progrès dans le domaine de l'industrialisation du lait, il peut être intéressant de passer en revue les réalisations qui semblent être les plus immédiates dans cette voie. Dans le domaine de l'étude des produits de réaction qui se produisent lors de la détérioration des produits laitiers, détérioration spontanée ou détérioration due aux enzymes, nos connaissances sont encore très restreintes et il est probable que l'intensification des recherches dans cette voie serait très profitable. Nous avons déjà souligné l'importance des phénomènes d'oxydation et de réduction et leur influence sur les qualités de conservation de certains produits laitiers. On a constaté que la présence de très faibles traces de groupes SH fortement réactifs empêche l'oxydation de la graisse contenue dans la poudre de lait, en présence d'air. 
Malheureusement, l'oxydation de ces groupes protecteurs contribue à faire apparaître un goût défectueux dans les produits conservés. Il apparaît done désirable de définir et de régler un état d'équilibre optimum entre les réactions d'oxydation et de réduction dont sont le siège les produits laitiers.

Le domaine de la chimie des enzymes s'étend rapidement. On a aujourd'hui fait la preuve expérimentale que plusieurs enzymes peuvent agir simultanément, ou successivement sur la caséine et sur la lactalbumine dans la molécule de laquelle se trouvent des lipides et il se peut que cette observation soit à la base de conséquences très importantes pour l'industrie du fromage. II n'est pas impassible en effet que par un emploi contrôlé des enzymes, on puisse transformer d'une façon révolutionnaire cette branche particulière de l'industrie laitière, et qu'on puisse même faire apparaître sur le marché des produits laitiers, des substances d'un caractère entièrement nouveau.

L'emploi du laetose en vue d'augmenter le rendement en pénicilline dans la fabrication de ce dernier produit, et la fabrication de l'acide lactique en partant du sérum de lait, sont deux exemples. qui montrent l'emploi que l'on peut faire de cet hydrate de carbone. Un autre exemple nous est donné également par la fabrieation d'un produit provenant du lactose et que l'on obtient par oxydation. électrolytique. Ce produit est constitué par un mélange de bromure de calcium et de lactobionate de calcium, il est utilisé comme agent sédatif et pour le traitement de certaines maladies nerveuses.

Dès aujourd'hui, d'importants développements sont en vue dans le domaine des applications de la caséine et de la lactalbumine pour la fabrication d'agents thérapeutiques et d'aliments spéciaux. Actuellement l'utilisation de la caséine pour cette application, est encore assez restreinte et limitée à quelques spécialités. Il semble que la caséine qui pourtant présente une grande valeur nutritive n'ait pas encore trouvé des applications qui lui reviennent. Les propriétés anti-acides de la caséine que l'on peut facilement mettre en évidence par les courbes de titrages potentiométriques n'ont pas encore attiré l'attention des spécialistes et il est certain que les médecins, lorsqu'ils connaîtront ce corps plus complètement, envisageront beaucoup plus volontiers son emploi. On peut par exemple prévoir que la caséine pourra être utilisée pour le traitement des ulcères peptiques. Néanmoins, il est certain que ces applications particulières se développeront, il sera nécessaire d'adopter pour la fabrication de la caséine, des procédés de fabrication beaucoup plus précis que ceux auxquels on a actuellement recours. Il est intéressant déjà dé citer dans cette voie la fabrication du caséinate 
dicalcique purifié ou dispersable, qui entre dans la préparation de certains produits alimentaires ou pharmaceutiques.

Mais il est probable que les hydrolysats de lactalbumine et de caséine seront peut-être encore plus utilisés que la lactalbumine et la caséine par l'industrie pharmaceutique, dès que leurs méthodes de production auront été améliorées. On cherche en particulier actuellement à retirer de ces substances par-adsorption sélective certains constituants qui en rendent le goût désagréable pour le consommateur. On a déjà utilisé une lactalbumine modifiée, facilement dispersable dans l'eau et présentant des propriétés hydrophiles extrêmement développées, au cours d'essais encore limités, pour le traitement d'œdèmes et de certaines malądies résultant d'un manque de protéines. Ce produit s'est également révélé efficace pour la régénération des protéines du sang et à ce point de vue se compare très favorablement au sérum du sang de bœuf. Des essais cliniques et nutritifs permettront certainement de montrer, dans un avenir proche, que la lactalbumine extraite du sérum, peut constituer un aliment et un produit pharmaceutique de première importance.

L'amélioration des propriétés nutritives du lait liquide par l'addition de concentrés de vitamine $\mathrm{D}$, se développe rapidement. Dans certains Etats des Etats-Unis, toutes les agglomérations de plus de 2.500 habitants, reçoivent déjà, pour la consommation humaine, un lait enrichi en vitamine D. Mais il est probable que cette technique d'enrichissement du lait se développera rapidement et les travaux en cours qui montrent le rôle physiologique capital de la vitamine A permettront de ne pas retarder plus longtemps l'enrichissement du lait vendu aux consommateurs en vitamine A ou en précurseur de eette vitamine.

En résumé, l'industrie laitière a bénéficié d'une façon considérable des recherches entreprises dans de nombreux domaines de la chimie pure. La chimie du lait et de ses dérivés se développe chaque jour et elle est à l'origine d'une conception' nouvelle de l'intérêt du lait à la fois comme matière première pour l'industrie chimique et comme aliment humain qui-peut et qui doit être enrichi ou modifié. 